\title{
La gastronomía como medio para el desarrollo de innovaciones sociales
}

\section{Gastronomy as a means for the development of social innovations}

\author{
Mariana Mejía-Rivas ${ }^{1}$ \\ Luz Guiomar Maldonado-Pérez ${ }^{2}$ \\ Recibido: octubre 29 de 2019 \\ Aceptado: mayo 15 de 2020
}

\section{Resumen}

El objetivo del trabajo fue el comprender las características de la innovación social, a partir de la gastronomía. La investigación tuvo un enfoque cualitativo y un diseño comprensivo. Se tuvo como unidad de análisis, las características de las innovaciones sociales dadas desde la gastronomía, y como unidad de comprensión: el concepto, método y validación de la innovación social realizada desde la gastronomía. Se realizó la selección de un corpus textual de 21 artículos, los cuales debían referirse a casos colombianos y trabajar en procesos de innovación social que incluyeran la gastronomía. La herramienta para el análisis de información fue Atlas.ti. Los resultados indican que, desde la gastronomía, pueden promoverse procesos de innovación social, y que las características atribuidas a la innovación social pueden ser también atribuidas a la innovación social desde la gastronomía. Se concluye que la gastronomía es una herramienta o medio para el desarrollo de innovaciones sociales, que puede generar cambios en las costumbres alimentarias de una comunidad; a la vez que una herramienta para un problema actual y relevante, como es la seguridad alimentaria.

Palabras clave: innovación social, gastronomía, seguridad alimentaria, cocina.

\begin{abstract}
This work presents the results of the research on characteristics of social innovation based on gastronomy. The research had a qualitative focus and a comprehensive design. The characteristics of the social innovations given from gastronomy were taken as the unit of analysis and as the unit of understanding the concept, method and validation of social innovation carried out from gastronomy. A choice was made from a textual corpus of 21 articles. which should refer to Colombian cases and work on social innovation processes that included gastronomy. The tool for the analysis of information was Atlas T. The results express that processes of social innovation can be promoted from gastronomy and that the characteristics attributed to social innovation can also be attributed to social innovation from gastronomy. It is concluded that gastronomy can be a tool or means for the development of social innovations that can generate changes in the eating habits of a community; as well as a tool for a current and relevant problem such as food security.
\end{abstract}

Keywords: social innovation, gastronomy, food security, kitchen.

1 Publicista, Magíster en Creatividad e Innovación en las Organizaciones, Universidad Autónoma de Manizales, Manizales, Colombia. E-mail: mmejiar@autonoma.edu.co. ORCID: 0000-0003-1148-4744

2 Licenciada en Filosofía y Letras, Magíster en Filosofía, Universidad Autónoma de Manizales, Manizales, Colombia. E-mail: Iguiomar@ autonoma.edu.co. ORCID: 0000-0001-9620-4398 


\section{Introducción}

Este proyecto, que busca comprender las características de la innovación social desde la gastronomía, parte del vacío conceptual relacionado con la Innovación social, en la cual la gastronomía ha sido utilizada para su logro. Si bien la gastronomía ha venido enriqueciéndose como campo de conocimiento en tanto ha trascendido a los espacios de la profesionalización, el vacío se da con relación al uso de la gastronomía en el campo de las innovaciones sociales.

Se encuentra que tanto a nivel nacional como internacional, existen proyectos que hacen uso de la gastronomía para el desarrollo de las innovaciones sociales. Ejemplo de ello es el trabajo de Mascarenhas y Gandara (2010), cuyo objetivo era realizar un análisis de la producción y transformación territorial, a través de la gastronomía como atractivo turístico. Asimismo, Mercado-Afanador y Rey-Patino (2015), en su trabajo buscaban fortalecer el turismo a partir de la innovación social, desarrollando productos gastronómicos que aporten soluciones sociales, a la vez que garantizan la seguridad alimentaria de la población de Cartagena de Indias. En Colombia también se encuentra el estudio de Villa y Melo (2015), cuyo objetivo fue la realización de un estado del arte sobre el impacto de la innovación social en el país.

A nivel internacional, se puede citar el trabajo de Jaeger y Erbe (2015), quienes señalan que las innovaciones sociales tienen un alto potencial para promover el desarrollo sostenible, así como la transformación de prácticas de consumo más sostenibles. El trabajo de Ortiz y Raquel (2015), un estudio comparativo, con el propósito de establecer el Insights del Chocolate Orgánico, en el Distrito Metropolitano de Quito. También se encuentra el trabajo de Ademola y Adende (2016), cuyo objetivo era reorientar las estrategias de desarrollo agrícola del continente africano, promoviendo: un crecimiento económico rápido y sostenible, la seguridad alimentaria y la eliminación de la pobreza en África. Por su parte, Martin y
Upham (2016), publicaron un modelo conceptual para determinar la movilización de los valores ciudadanos a través de innovaciones de base, desde la teoría de valor de Schwartz y la teoría sobre la promulgación colectiva de valores.

Es importante entender la gastronomía como una práctica inherente a todas las culturas humanas, desde la antigüedad hasta la actualidad (AcleMena, Santos-Díaz \& Herrera-López, 2020). Esta ha generado espacios de socialización y sociabilidad de las comunidades, desarrollando hábitos de alimentación, pero más allá de ello, espacios en los que se pueden generar lazos entre los miembros de las comunidades. Además, la gastronomía se ha convertido en un campo de conocimiento que recibe aporte de ciencias como: la química, la física o la biología, así como de las ciencias sociales y humanas (Reyes-Uribe, Guerra-Avalos \& Quintero-Villa, 2017).

En los siguientes apartados se expone el marco teórico, resaltando las principales teorías que se recogieron con respecto a la innovación social. Luego se expone la metodología, señalando las categorías y subcategorías trabajadas para analizar los resultados. Posteriormente, se efectúa la discusión y se presentan las conclusiones del estudio.

\section{Marco teórico y metodología}

\subsection{Sobre el concepto de innovación}

Aunque hay distintas definiciones acerca de la innovación, en todas ellas existe un elemento en común: la innovación implica la generación de ideas y la materialización de estas en productos, bienes o servicios. A continuación, se presentan algunas definiciones de innovación, aclarando que no se encontraron textos que hablaran específicamente de la innovación social desde la gastronomía, como un campo de conocimiento o de aplicación. En este sentido, se llevó a cabo una revisión general de las teorías de la innovación social. 
Rogers (1962), indica que: "an innovation is an idea, practice, or object that is perceived as new by an individual or other unit of adoption". Esta definición, tiene su base en la idea de novedad, aspecto del significado que ha atravesado gran parte de la del desarrollo histórico de la innovación. Por su parte, Visser (2002), presenta la agricultura como la primera innovación realizada por la humanidad, en la medida en que esta generó cambios en las prácticas alimentarias. Luego vendrían una gran cantidad de desarrollos innovadores, como la electricidad y la bombilla; sin embargo, con el surgimiento de la ciencia moderna, se ha presenciado un desarrollo más acelerado de la innovación en nuestro mundo, la cual está vinculada a una amplia capacidad de intervenir en sus leyes. Esto también se relaciona con: la determinación de las estrategias, métodos y las prácticas para la creación de ideas, productos o servicios novedosos, así como a la generación de conocimiento nuevo. No obstante, la velocidad con la que se producen dichos cambios, ha llevado a la obsolescencia de algunas ideas, productos o servicios y, por lo tanto, cada vez se hace más necesario innovar.

Arboníes (2006), vinculó a la idea de innovación la de gestión del conocimiento, señalando que: un concepto universal y fundamental dentro y para el desarrollo de la innovación, es el conocimiento. Este autor también indica que: "no necesitamos más optimización de recursos, sino recuperar el discurso sistémico, humano, social y contextualizado que cuestione y reconstruya el pensamiento dominante, y nos ayude a entender la innovación, la creatividad como clave de evaluación en las organizaciones". Igualmente, se hace énfasis en la importancia de los individuos que conforman el colectivo, en tanto la "innovation is people process" (Rotwell, como se citó en Arboníes, 2006).

Karl Sveiby, citado en Arboníes (2006), explica que "la principal fuente de ventaja competitiva reside en la innovación continua y esta se consigue a través de la creación del conocimiento" (p. 214). Asimismo, en cuanto a "Innovación y gestión del conocimiento", Carballo (2006), indica que: "La escasez no es más que la condición necesaria para la innovación; solo si es acompañada de una idea, de un sentido, del sentido común, de un proyecto, de una estrategia" (p. 4). En este sentido, se pone de frente una condición específica para promover el desarrollo de las innovaciones.

En el Manual de Oslo, la innovación se define como:

(...) la introducción de un nuevo, o significativamente mejorado producto (bien o servicio), de un proceso, de un nuevo método de comercialización o de un nuevo método organizativo en las prácticas internas, de la empresa, la organización del lugar de trabajo o las relaciones exteriores. (Echeverría, 2008, p. 56)

Aunque en este manual la innovación se define desde el marco organizacional, es de resaltar su universalidad, y el concepto de "mejora significativa", que nos conduce a pensar que la innovación no solo es novedad, sino es además perfeccionamiento o cambio.

2.2 Delimitación del concepto y alcance de la innovación social

En tanto los estudios acerca de la innovación social son relativamente nuevos, aún se encuentran vacíos semánticos que dificultan la delimitación y socialización de lo que es la innovación social. No obstante, según la revisión realizada, el término surgió en el siglo pasado. Encontramos entonces que, durante "la década de los sesenta, pensadores como Peter Drucker - un referente indiscutible del management-, Michael Young -fundador de la Open University- y los escritores franceses Pierre Rosanvallon, Jacques Fournier y Jacques Attali, hayan aportado a su estudio (Chambon, David \& Devevey, 1982). La innovación social puede entenderse como: un proceso que tiene como propósito la generación de valor social, por medio de prácticas, modelos de gestión, productos o servicios novedosos que satisfacen necesidades, 
aprovechan oportunidades y resuelven problemas de forma más eficiente y eficaz que otros, produciendo un cambio estructural favorable en el sistema en operación (Departamento Nacional de Planeación, 2013).

Echeverría (2008), destaca otro elemento importante sobre la innovación social, el del interés social:

(...) La innovación social ha de referirse a los valores sociales, por ejemplo, el bienestar, la calidad de vida, la inclusión social, la solidaridad, la participación ciudadana, la calidad medioambiental, la atención sanitaria, la eficiencia de los servicios públicos o el nivel educativo en la sociedad. (p. 610)

Asimismo, para Easteal (2013), las innovaciones sociales corresponden a: nuevas ideas, productos, servicios y modelos, que tienen como propósito la satisfacción de necesidades, e implican las relaciones y/o colaboraciones sociales. Abreu-Quintero (2011), quien realizó un estudio acerca de las teorías de la innovación social, señala la importancia de orientar estas innovaciones para que aporten: al desarrollo y promoción de los valores sociales, a la economía, al desarrollo social en general, a la productividad y a la competitividad de las empresas.

En su trabajo, Abreu-Quintero (2011), referenciando a Murray, Mulgan y Caulier, señala que la innovación social puede cambiar tanto al Estado como al mercado, con la finalidad de contribuir a la mejora de condiciones sociales tanto individuales como grupales; sin embargo, en la innovación social no solo intervienen el Estado y el mercado, también lo hace el hogar y la macroeconomía, de manera que estos cuatro aspectos deben ser tenidos en cuenta de manera interrelacionada. La importancia de esta consideración, se da en tanto que la innovación social no depende solo de un sector específico.
Mulgan, Tucker, Rushanara y Sanders (2007), señalan que la"Social innovation refers to new ideas that work in meeting social goals". Ellos ponen el acento en que las innovaciones sociales pueden ser desarrolladas y difundidas por organizaciones que tienen propósitos sociales.

En el informe de Buckland y Murillo (2014), se señala que las innovaciones sociales tienen un carácter social de principio a fin, y que los beneficios que generan abarcan a quienes las implementan, las replican, así como a las comunidades. Es importante resaltar que no todas las innovaciones sociales generan beneficios económicos, hay innovaciones que generan beneficios culturales, cambios en prácticas ancestrales transmitidas generacionalmente o reordenamiento de estructuras sociales, entre otras.

En síntesis, se puede decir que la innovación social, puede ser: un proceso, una idea, un producto o un servicio, que tiene por objeto cubrir o satisfacer necesidades o resolver problemas de las comunidades.

\subsection{Aproximación al concepto de gastronomía}

La gastronomía es el conocimiento razonado de todo lo que se relaciona con el hombre, en lo que a su alimentación se refiere (Gutiérrez, 2012). El concepto de gastronomía se encuentra en estrecha relación con dos aspectos cotidianos de la vida humana: la cultura de la alimentación y el acto mismo de la alimentación. Cecilia Isabel Gutiérrez de Alba (2012), en su libro "Historia de la gastronomía”, expresa que: "La gastronomía rige toda nuestra vida. Así como el llanto de un recién nacido busca el seno materno para alimentarse, el moribundo busca el alimento espiritual antes de partir".

Este hecho abarcante sobre nuestras vidas, ha puesto cada vez más a la gastronomía como un objeto de estudio que busca responder no solo a los aspectos sociales y culturales que la rodean, sino también a la crisis alimentaria cada vez más 
urgente para la humanidad. Ello ha implicado que su estudio haya influido en la transformación de los métodos de producción de toda la cadena alimentaria, así como en la transformación de las prácticas alimentarias ancestrales, permitiendo la generación de políticas públicas de carácter social y medioambiental. Es importante reconocer que la gastronomía es un campo de conocimiento que se construye desde la multidisciplinariedad, donde las ciencias básicas y las ciencias sociales y humanas contribuyen a su construcción.

(...) La búsqueda de raíces gastronómicas, así como la forma de cómo entender la cultura de un lugar por medio de su cocina, están adquiriendo cada vez mayor importancia. La cocina tradicional está siendo reconocida como un componente valioso del patrimonio intangible de los pueblos. (Nunes, 2007, p. 239)

Desde la perspectiva de las ciencias básicas, vemos cómo la cocina nuclear ha alcanzado un fuerte auge en la actualidad, a partir del conocimiento de la física y química de los alimentos, y los procesos a que estos son sometidos (Casas-Mateus, Albarracín-Tunjo \& Cortés-González, 2017). Pero el estudio físico-químico de los alimentos, no solo contribuye al desarrollo de productos gastronómicos novedosos, también permite, por ejemplo, un conocimiento de estos desde sus propiedades nutricionales.

\subsection{Metodología}

La investigación se realizó con un enfoque cualitativo, donde la interpretación implicó una aproximación a textos de innovación social desde la gastronomía, contrastados a la luz de las teorías sobre la innovación social. Esto permitió realizar generalizaciones inductivas de carácter interpretativo, acerca de las características de la innovación social desde la gastronomía. Se trabajó con un análisis comprensivo de dieciséis textos, que dan cuenta de la inclusión e innovación en gastronomía. Para ello, se tomó como unidad de análisis a las características de las innovaciones sociales dadas desde la misma; mientras que la unidad de comprensión fue: el concepto, método y validación de la innovación social desde la gastronomía.

Las categorías analizadas se tomaron de Buckland y Murillo (2014), quienes expresan como características de la innovación social: el impacto social, la sostenibilidad económica, el tipo de innovación, la colaboración intersectorial y la escalabilidad y replicabilidad. Las subcategorías emergentes, surgieron del análisis comprensivo de las definiciones de las categorías iniciales y del análisis de los textos. La información se registró mediante el análisis sistémico de texto, que mostraba información sobre innovación social en el área en cuestión. Se elaboraron fichas que identificaban cada uno de los textos, que se sometieron a análisis textual comprensivo, con la herramienta ATLAS.ti versión 1.6.0.

Para la presentación de resultados y el análisis, se hizo uso de la teoría de soporte de cada una de las categorías, la cual surge de acuerdo con las necesidades entregadas en la revisión de las categorías. Para ello se tuvo en cuenta lo expresado por, Hernández-Sampieri, Fernández-Collado y Baptista-Lucio (2006):

(...) al finalizar el análisis y elaborar el reporte cualitativo, el investigador debe vincular los resultados con estudios anteriores, esto es con el cuerpo del conocimiento que se ha generado respecto al planteamiento del problema. (...) Así mismo, algunos descubrimientos pueden ser soportados por la literatura (Creswel, 2000). Pero, finalmente, el grado en que se incluya a la literatura en el reporte, es un asunto sobre el cual cada investigador hará su propio juicio y tomará su decisión. (p. 729)

\section{Resultados y discusión}

En cada uno de los textos revisados, se encontraron al menos 3 de las categorías analizadas. Esto debido, posiblemente, a que los textos no 
estaban exponiendo estas innovaciones desde una perspectiva teórica de la innovación social, sino presentando los aspectos más relevantes de experiencias de innovación social. En la figura 1, se presentan las categorías y subcategorías del estudio.

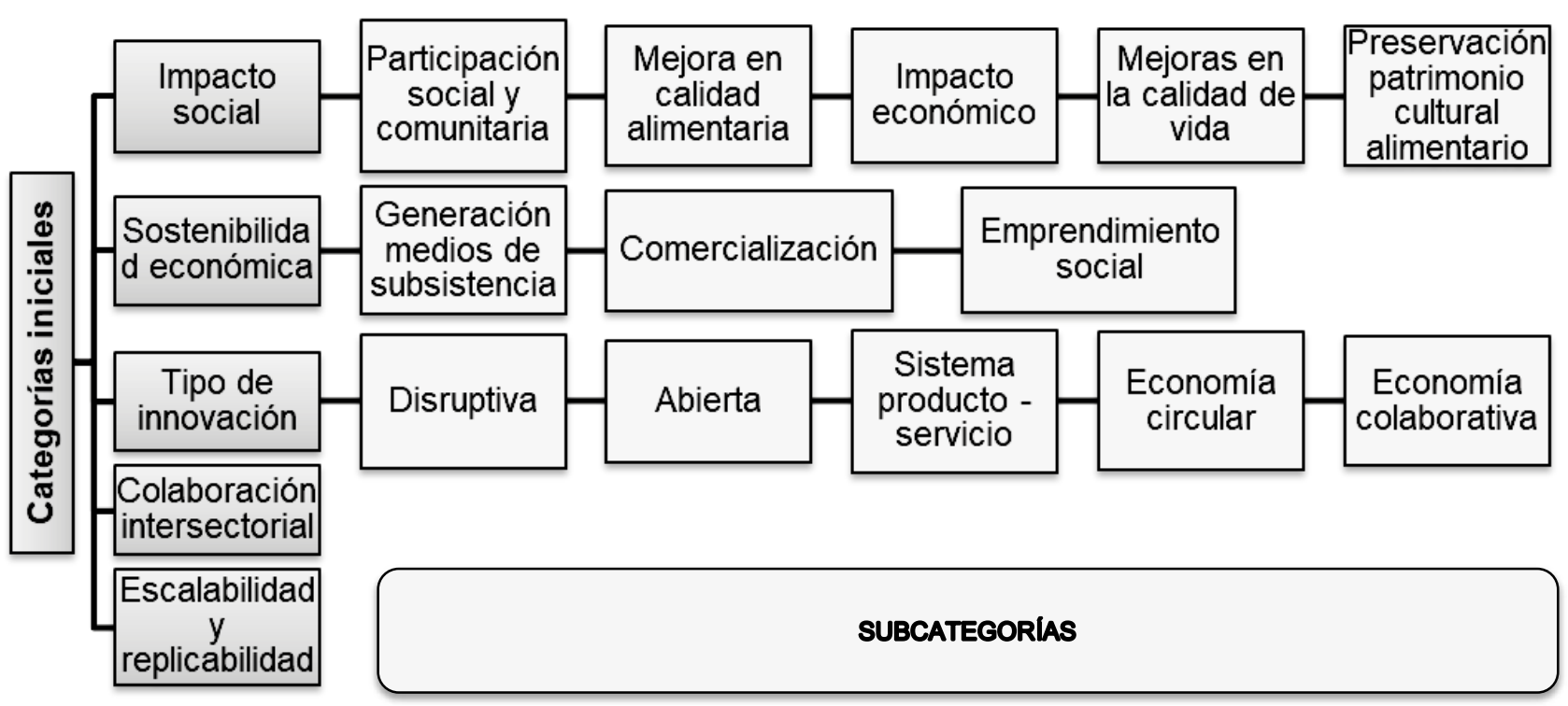

Figura 1. Categorías y subcategorías analizadas.

La categoría de impacto social tiene como propósito principal, el de lograr mejoras en la calidad de vida. Para ello, es importante la participación social y comunitaria, que implica que las comunidades o grupos sociales se involucren en el proceso de innovación, a partir de objetivos comunes que buscan la solución a: problemáticas sociales, económicas, políticas y culturales, entre otras. En este sentido, la participación social comunitaria implica que:

(...) La apropiación del proceso se consolida en la medida en que las comunidades que han participado en la metodología, están en capacidad de compartir el aprendizaje con otras comunidades, así como liderar réplicas de menor escala en sus regiones. (Gutiérrez, 2010, p. 527)
Por su parte, las Mejoras en la Calidad de Vida pueden evidenciarse a través de: "un estado de satisfacción general, derivado de la realización de las potencialidades de la persona" (Ardila, 2013). Esta sensación de satisfacción puede ser de carácter tanto subjetivo como objetivo, e implica aspectos físicos, emocionales y sociales. Ejemplo de esta subcategoría, es el Proyecto Social Necoclí, que tenía como objetivo:

(...) Fortalecer el componente educativo y los procesos de formación y producción de cacao, para contribuir al mejoramiento de la calidad de vida de la población, en la zona de influencia del proyecto productivo. (Fundación Luker, 2017, p. 49)

La subcategoría: mejora en la calidad de vida alimentaria, se puede describir como las buenas 
prácticas alimentarias para mejorar la nutrición de las personas o comunidades, así como también en las buenas prácticas de la producción de alimentos que conducen a la seguridad alimentaria. El proyecto de Mercado-Afanador y Rey-Patiño (2015), muestra cómo la innovación social puede impactar de manera positiva, tanto a la agricultura como al sector turístico, a través del desarrollo de recetas gastronómicas de regiones y ciudades como Cartagena de Indias. Este impacto puede generar nuevas opciones para el consumo y aprovechamiento de los alimentos, lo cual afecta de manera directa las costumbres alimentarias de las poblaciones nativas, así como de los turistas. Esta subcategoría es importante en tanto es un elemento propio de la innovación social, dada desde la gastronomía, ya que se hace impacto directamente sobre aspectos gastronómicos.

La subcategoría de impacto económico, se presenta cuando se mejoran las condiciones económicas de las personas a través de actividades relacionadas con la producción y comercialización de productos, en este caso gastronómicos. Ejemplo de esto, es:

(...) Comproagro, una plataforma tecnológica que elimina los intermediarios en el proceso de compra y venta de productos agrícolas y un centro de acopio para los agricultores de Boyacá, que reúne a pequeños campesinos para vender productos en masa a grandes superficies. (Avellaneda, 2017, p. 2)

La subcategoría: preservación del patrimonio cultural alimentario, implica el conocimiento de las tradiciones alimentarias. A partir de allí, se generan innovaciones que permitan la potenciación de los productos gastronómicos tradicionales de las comunidades. Reconocer esta tradición, implica la comprensión identitaria de las comunidades. Con el proyecto de la Fundación Leo, se pretendía:

Reforzar el uso del arroz como materia prima y su consumo con el fin de generar empoderamiento en esta comunidad, por medio de un laboratorio creativo que aportara a la consolidación de procesos sociales y económicos, utilizando los ingredientes locales. (Fundación Leo Espinosa, s.f)

Referente a la categoría Impacto social, se encontró que esta responde, desde sus subcategorías, a lo propuesto por Libera-Bonilla (2007), y está estrechamente relacionada con la idea de cambio que manifiesta este autor. El elemento novedoso de esta categoría, corresponde a la subcategoría de la preservación del patrimonio cultural alimentario, la cual podemos considerar como propia de la innovación social dada desde la gastronomía.

La categoría de sostenibilidad económica, busca la generación de prácticas con impacto en las economías individuales y grupales con la finalidad de generar mejoras en la calidad de vida. En esta categoría, emergieron 5 subcategorías. La primera, generación de medios de subsistencia, consiste en el desarrollo de actividades que generen activos para una subsistencia sostenible en el tiempo. Ejemplo de ello es el trabajo de Mercado-Afanador y Rey-Patiño (2015), que pretendía:

(...) Direccionar esfuerzos hacia la seguridad alimentaria, en la correcta gestión de los abundantes y diversos recursos agrícolas con los que cuenta la región, provocará, sin lugar a duda, un impacto positivo sobre el sector agropecuario. (p. 134)

La subcategoría comercialización, implica el desarrollo, planeación y realización de actividades que permitan llevar los productos gastronómicos al mercado, para darlos a conocer y ser vendidos. Un caso exitoso de comercialización, es el de FresOta, donde los productores suprimieron la intermediación para la comercialización del producto; además, determinaron políticas de pago a socios y proveedores, lo cual generó cambios en la estructura de costos y la apertura de nuevos mercados. Esto se refleja en que: “En Cali se logran alianzas formales, expresadas en contratos con los almacenes de grandes superficies y otros distribuidores" (Camacho-Velásquez, s.f, p. 50). 
Otra subcategoría es El emprendimiento social, con la cual se busca, desde el liderazgo social, dar solución a problemas sociales. En un informe de Casa Luker, la fundación muestra los impactos generados en la sociedad desde diferentes tipos de proyectos, incluidos los gastronómicos. Se ha logrado llegar a comunidades afectadas por el conflicto armado, las cuales erradicaron los cultivos ilícitos y los reemplazaron con el cacao (Fundación Luker, 2017).

Se puede afirmar que todas las categorías correspondientes a la sostenibilidad económica, se encuentran en las innovaciones sociales dadas desde la gastronomía. Desde esta perspectiva, se puede afirmar que los hallazgos son coherentes con lo referenciado en el marco teórico. Es de anotar que el emprendimiento, necesita de la colaboración intersectorial, lo cual se encuentra en los casos revisados; además se evidencia la importancia de los emprendimientos como motor de desarrollo empresarial, social y cultural.

La categoría Colaboración Intersectorial, señala que: "La Innovación Social es un modelo que permite construir nuevas formas de relación entre la sociedad civil y las instituciones, para la generación de valor público compartido" (Domanski, Monge \& Quitianez, 2016, p. 24). Esta subcategoría, muestra la importancia de las alianzas entre diferentes actores para el desarrollo de la innovación, en tanto se aúnan esfuerzos y fortalezas que permiten garantizar el éxito de la misma. En los trabajos revisados, se encuentran: alianzas Estado-empresa- organizaciones, academia-sociedad civil, Estado-Ongs, entre otras. Ejemplo de ello son las huertas caseras, en las cuales hay asociación entre los padres, comunidad y agentes educativos comunitarios e institucionales; todos trabajan asociativamente para la producción y comercialización de alimentos, lo que conduce a la "vinculación de las comunidades a las mesas de articulación y concertación" (Instituto Colombiano de Bienestar Familiar, 2010, p. 21).
Para la categoría tipos de innovación, se tomaron las subcategorías de: innovación disruptiva, abierta, producto servicio, economía circular y economía colaborativa.

La Innovación disruptiva, es un "tipo de innovación que ayuda a crear nuevos mercados y cadenas de valor y que, eventualmente, reemplaza la tecnología anterior con nuevos productos o servicios no esperados por los mercados" (Buckland \& Murillo, 2014, p. 60). Un ejemplo de este tipo de innovación lo constituye Comproagro, plataforma tecnológica mencionada anteriormente (Avellaneda, 2017).

La innovación abierta: "asume que las organizaciones deberían utilizar ideas externas e internas para avanzar en sus productos y servicios, donde el flujo de conocimiento puede ser hacia la empresa o hacia sus grupos de interés externos" (Buckland \& Murillo, 2014, p. 37). Esta innovación implica la apertura de las comunidades, para generar y recibir ideas de otros sectores. Un ejemplo de este tipo de innovación, se observa en la implementación de la metodología Innovación Rural Participativa en la zona andina central de Colombia, donde la academia, a través de sus investigadores, realizó un trabajo de participación con las organizaciones de productores y la comunidad:

(...) Mediante el trabajo colectivo, se identifican las cadenas productivas donde los grupos pueden participar con los productos de innovación derivados de las investigaciones. A partir de este proceso, se contactan aliados y se formulan proyectos conjuntos. (Gutiérrez, 2010, p. 530)

La subcategoría de producto o servicio, corresponde a un "Modelo de negocios que ofrece soluciones para satisfacer necesidades a través de una combinación de servicios y productos, que en muchos casos consiste en la desmaterialización y la reducción del consumo" (Buckland \& Murillo, 2014, p. 38). Con relación a esta subcategoría, no se encontró un ejemplo claro de la misma en el material revisado. 
La subcategoría de economía circular, se refiere a una clase de economía que nace con el fin de buscar diferentes soluciones en el uso de todo tipo de residuos, que afectan al medio ambiente. El caso de las huertas caseras es un ejemplo de esta economía, en tanto se hace reutilización de la materia orgánica; una de las prácticas de la comunidad consiste en: "la preparación de abonos compuestos, a partir de los desechos de la cocina, papel y otros materiales orgánicos" (Instituto Colombiano de Bienestar Familiar, 2010, p. 5). Esto no solo permite dar solución al manejo de residuos, sino que también permite la disminución de costos en la inversión de nutrientes para los cultivos.

Con respecto a los tipos de innovación, se observa que las innovaciones sociales dadas desde la gastronomía, pueden ser: abiertas, disruptivas o de producto servicio, lo cual es coherente con la teoría que se refiere a los tipos de innovación.

La economía colaborativa, según Buckland y Murillo (2014), está "basada en la redistribución de poder desde las instituciones centralizadas hacia las personas y las comunidades, en la cual el consumidor llega a ser coproductor, co-creador y redistribuidor" (p. 39). Un ejemplo de economía colaborativa, lo constituye la experiencia de FresOta, ya que esta asociación apoyada por entidades, como: el SENA, la alcaldía municipal de Sotará y la Fundación Smurfit Cartón de Colombia, permiten la apropiación de la cooperativa por parte de la comunidad que la conforma.

En cuanto a las categorías de escalabilidad y replicabilidad, se encuentra que: la primera implica la apertura de nuevas filiales, mientras que la replicabilidad señala la transferencia de las innovaciones sociales a otros grupos sociales. Es difícil separar la escalabilidad de la replicabilidad, casi se podría decir que hay cierta interdependencia entre ellas. Ejemplos de innovaciones que fueron replicables y escalables, se encuentran en muchos de los trabajos de la Fundación Leo, donde las prácticas realizadas a partir de pequeñas comunidades pueden ser llevadas a otras, a través de procesos formativos.

Otro ejemplo es el caso del Interno, experiencia Suiza retomada por Johana Bahamón, que es un trabajo:

(...) en torno a un propósito común, al crear una novedosa forma de resocialización, materializada en esta iniciativa única, abriendo las puertas de una cárcel para brindar un servicio de restaurante de la mejor calidad y lograr para las internas una segunda oportunidad, para reinsertarse en el tejido social y productivo. (Restaurante Interno, s.f)

\section{Conclusiones}

Esta investigación tuvo como objetivo comprender las características de la Innovación Social a partir del análisis de trabajos publicados sobre innovaciones sociales, en el campo de la gastronomía. Se encontró que las categorías de análisis iniciales que pueden dar cuenta de las innovaciones sociales, están poco desarrolladas teóricamente. Es decir, se encuentran definiciones básicas de las mismas, por lo cual las categorías emergentes permitieron comprender a mayor profundidad el concepto de innovación social.

Las innovaciones sociales que hacen uso de la gastronomía, cumplen con los conceptos básicos de lo que es una innovación social, ya que en términos generales buscan la satisfacción de necesidades de las comunidades desde las categorías trabajadas, sin embargo, el factor diferenciador de una innovación social dada desde la gastronomía, se encuentra en los cambios alimentarios de las comunidades, con miras a favorecer aspectos nutricionales y de seguridad alimentaria. Esto se une a la preservación del patrimonio cultural alimentario, como elemento identitario fundamental de las comunidades. 
Se concluye que para el desarrollo de las innovaciones sociales, en especial las que se dan desde la gastronomía, es importante el uso de metodologías participativas, pues ellas promueven la participación comunitaria. En este sentido, se logra que las comunidades apropien las innovaciones, permitiendo su perdurabilidad en el tiempo.

\section{Agradecimientos}

Artículo producto del informe final de investigación: Características de la innovación social desde la gastronomía, para optar al título de Magíster en Creatividad e Innovación en las Organizaciones, de la Universidad Autónoma de Manizales. Tesis sustentada en agosto de 2019.

\section{Referencias}

Abreu-Quintero, J. (2011). Innovación social: conceptos y etapas. Daena. International Journal of good Conscience, 6 (2), 134-148.

Acle-Mena, R. S., Santos-Díaz, J. Y., \& Herrera-López, B. (2020). La gastronomía tradicional como atractivo turístico de la ciudad de puebla, México. Revista de Investigación, Desarrollo elnnovación, 10(2), 237-248. doi: 10.19053/20278306.v10.n2.2020.10624

Ademola, A., \& Adenle, L. M. (2016). Agribusiness innovation: A pathway to sustainable economic growth in Africa. Trends in Food Science \& Technology, 17. doi: 10.1016/j.tifs.2016.11.008

Arboníes, A. L. (2006). Conocimiento para innovar. Cómo evitar la miopía en la gestión del conocimiento. Madrid, España: Ediciones Díaz de Santos S.A.

Avellaneda, J. (2017). Joven crea aplicación que elimina intermediarios en el campo. Innovación social, 1-5.

Buckland, H., \& Murillo, D. (2014). La innovación social en América latina: Marco conceptual y agentes.
Barcelona, España: Fondo Multilateral de Inversiones e Instituto de Innovación Social de ESADE.

Camacho-Velásquez, J. (s.f.). Una experiencia muy jugosa. RedEAmérica. Recuperado de: http:// www.redeamerica.org/Portals/0/BuenasPracticas/Transformadores/FresOta_Cronica_ESP. pdf?ver=2018-03-12-095943-110.

Carballo, R. (2006). Innovación y gestión del conocimiento. España: Díaz de Santos.

Casas-Mateus, J., Albarracín-Tunjo, l., \& Cortés-González, C. (2017). Gastronomía molecular. Una oportunidad para el aprendizaje de la química experimental en contexto. Tecné, Episteme y Didaxis, $42,125-142$.

Chambon, J. L., David, A., \& Devevey, J. M. (1982). Les innovations sociales. Paris, France: Presses universitaires de France.

Domanski, D., Monge, N., \& Quitianez, D. (2016). Innovación social en Latinoamérica. Bogotá, Colombia: Corporación Universitaria Minuto de Dios.

Departamento Nacional de Planeación, Departamento Administrativo de Ciencia, Tecnología e Innovación y Agencia Nacional para la Superación de la Pobreza Extrema. (2013). Bases Conceptuales para una política de innovación social. Recuperado de: http://repositorio.colciencias.gov.co/ handle/11146/285

Easteal, S. (2013). How can social innovation help reduce food waste?.Fusions. Recuperado de: https:// www.eu-fusions.org/index.php/download?download=9:how-can-social-innovation-help-reduce-food-waste.

Echeverría, J. (2008). Manual de Oslo y la innovación social. ARBOR, 184 (732), 609-618. 
Fundación Leo Espinosa. (s.f.). Fortalecimiento del aprovechamiento y el consumo del arroz como ingrediente estratégico para la comunidad nayera. Recuperado de: http://funleo.org/portfolio-item/ fortalecimiento-del-aprovechamiento-y-consumo-del-arroz-en-el-rio-naya/.

Fundación Luker (2017). Reporte de sostenibilidad 2017. Recuperado de: http://fundacionluker.org. co/wp-content/uploads/reportesSostenibilidad/ reportesostenibilidad2017/es/CasaLuker.html

Gutiérrez, O. (2010). Desarrollo de una metodología rural participativa en la zona andina central de Colombia. Agronomía Colombiana, 28 (3), 525-533.

Gutiérrez, I. (2012). La historia de la gastronomía. Tlalneplanta, México: Red Tercer Milenio.

Hernández-Sampieri, R., Fernández-Collado, C., \& Baptista-Lucio, M. (2006). Metodología de la investigación. México McGraw-Hill.

Instituto Colombiano de Bienestar Familiar. (2010). La huerta familiar es una estrategia para promover el ejercicio de una alimentación saludable. Bogotá, Colombia: ICBF.

Jaeger, M., \& Erbe, J. R. (2015). Sustainable consumption through social innovation: a typology of innovations for sustainable consumption practices. Journal of cleaner Production, 784-798.

Libera-Bonilla, B. E. (2007). Impacto, impacto social y evaluación del impacto. ACIMED, 15 (3), 1024-9435. Recuperado de: http://scielo. sld.cu/scielo.php?script=sci_abstract\&pi$\mathrm{d}=\mathrm{S} 1024-94352007000300008 \& \mathrm{lng}=\mathrm{es} \& \mathrm{nrm}=\mathrm{iso}$.

Ortiz, L., \& Raquel, C. (2015). Insigths de chocolate orgánico en el mercado ecuatoriano: Caso comparativo "Pacari y República del cacao. Recuperado de: http://repositorio.puce.edu.ec/handle/22000/9236.
Martin, C. J., \& Upham, P. (2016). Grassroots social innovation and the mobilization of values in collaborative consumption: a conceptual model. Journal of Cleaner Production, 204-213. Doi: https://doi. org/10.1016/j.jclepro.2015.04.062

Mascarenhas, R. G., \& Gandara, J. M. (2010). Producción y transformación territorial: La gastronomía como atractivo turístico. Estudios y perspectivas en turismo, 19, 776-791.

Mercado-Afanador, P., \& Rey-Patiño, D. (2015). Turismo y desarrollo comunitario: La innovación social y seguridad alimentaria como base de desarrollo del producto gastronómico. Revista Methodos, 13 (13), 109-116.

Mulgan, G., Tucker, S., Rushanara, A., \& Sanders, B. (2007). Social Innovation. Oxford, Inglaterra: The Young Foundation.

Nunes, C. (2007). Somos lo que comemos: Identidad cultural, hábitos alimenticios y turismo. Estudios y Perspectivas en Turismo, 16 (2), 234-242. Restaurante Interno. (s.f.). Recuperado de: http://restauranteinterno.com.

Reyes-Uribe, A. C., Guerra-Avalos, E. A., \& Quintero-Villa, J. M. (2017). Educación en gastronomía: su vínculo con la identidad cultural y el turismo. El periplo sustentable, (32), 00009.

Villa, L., \& Melo, J. (2015). El panorama actual de la innovación social en Colombia. Banco Interamericano de Desarrollo. Recuperado de: https://publications.iadb.org/bitstream/handle/11319/6957/CTI_ DP_Panorama_actual_de_la_innovacion_social. pdf? sequence $=1$.

Visser, J. (2002). Innovación: Necesidad científica y elección artística. Recuperado de: http://www.learndev.org/dl/Innovacion-UdG-2002.pdf. 
